\title{
Free Vibration Analysis of Functionally Graded Plates FG with Porosities
}

\author{
Merdaci Slimane ${ }^{1,2 *}$, Belmahi Samir ${ }^{2}$, Belghoul Hakima ${ }^{1}$, Hadj Mostefa Adda $^{3}$ \\ ${ }^{1}$ University of Sidi Bel Abbes, Faculty of Technology, Civil Engineering and Public Works Department, Algeria. \\ ${ }^{2}$ University of Sidi Bel Abbes, Faculty of Technology, Department of Civil Engineering and Public Works, S, Algeria. \\ ${ }^{3}$ Centre universitaire de Rélizane, Département de Génie Civil, Institut des Sciences \& Technologie, Rélizane, Algeria.
}

\begin{abstract}
This article presents the free vibration analysis of simply supported plate FG porous using a high order shear deformation theory. In is work the material properties of the porous plate FG vary across the thickness. The proposed theory contains four unknowns unlike the other theories which contain five unknowns. This theory has a parabolic shear deformation distribution across the thickness. So it is useless to use the shear correction factors. The Hamilton's principle will be used herein to determine the equations of motion. Since, the plate are simply supported the Navier procedure will be retained. To show the precision of this model, several comparisons have been made between the present results and those of existing theories in the literature for non-porous plates. Effects of the exponent graded and porosity factors are investigated.
\end{abstract}

Keywords: Composite material; plates $\mathrm{FG}$; shear deformation theory; high order theory; free vibration; porosity.

\section{INTRODUCTION}

Composite materials are known as the modern materials which are composed of two or more different materials, to have the desired properties in specified applications. The lightweight composite materials known as fiber-matrix laminated composites have been used successfully in aircraft, automotive, marine industries and other engineering applications. However, the mismatch in mechanical properties across the interface of two different materials may cause large inter-laminar stresses. Consequently, de-bonding and delimitation problems can occur, especially in a high temperature environment.

To remedy such defects, functionally graded materials (FGMs), within which material properties vary continuously, have been proposed. The concept of FGM was proposed in 1984 by a group of materials scientists, in Sendai, Japan, for thermal barriers or heat shielding properties [1].

However, in the manufacture of FGMs, micro-porosities or voids can occur in the materials during the sintering process. This is due to the large difference in solidification temperature between the material constituents [2]. Wattanasakulpong et al. [3] also gave a discussion of the porosities that occur within FGM specimens made by a sequential multi-step infiltration technique. Therefore, it is important to take into account the effect of porosity in the design of FGM structures subjected to static [9-10] and dynamic loads [4]. Consequently, studies devoted to understanding the static and dynamic behavior of FGM material structures have been given more and more attention in recent years.

This work aims at developing a new simple theory of high order shear deformation for the analysis of the free vibration of FG plates by considering the porosities that can occur inside the materials with gradient properties (FG) at during their manufacture. The proposed theory contains four unknowns unlike the other theories which contain five known at most. Analytical solutions are obtained for the FG pallet by the present theory. The effects of various parameters, such as thickness ratio, gradient index, and volume fraction of porosity on the free vibration of plates FG are all discussed.

\section{MATHEMATICAL MODEL}

Consider a thick rectangular plate FG of length a, width $\mathrm{b}$ and thickness $\mathrm{h}$ made of functionally graded material as shown in Fig.1 together with the adopted coordinate system. The material properties of the plate FG, such as Young's modulus E, are assumed to be function of the volume fraction of constituent materials. The properties of the FGM vary continuously due to the progressive volume fraction of the constituents of the materials (ceramic and metal), generally in the direction of the thickness. The function of the P-FGM power law is commonly used to describe these variations in the properties of materials. The expression given below represents the profile of the volume fraction.

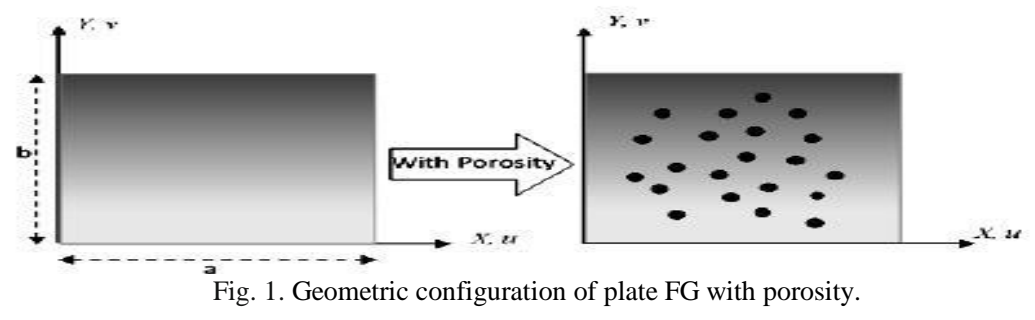


A plate FG made from a mixture of two materials, for example, metal and ceramics. The properties of the plate FG material are assumed to be continuously varied across the thickness of the plate. In this study, the imperfect plate is assumed to have porosities distributed in thickness due to defect during production. Consider an imperfect FG with a volume fraction of porosity, $\alpha,(0 \leq \alpha \leq 1)$ distributed uniformly between metal and ceramic, the law of the modified mixture proposed by Wattanasakulpong and Ungbhakorn [24] is used as:

$$
\begin{array}{ll}
E(z)=E_{m}\left(1-V-\frac{\alpha}{2}\right)+E_{c}\left(V-\frac{\alpha}{2}\right), \text { and } & V=\left(\frac{1}{2}-\frac{z}{h}\right)^{p} \\
\rho(z)=\rho_{m}\left(1-V-\frac{\alpha}{2}\right)+\rho_{c}\left(V-\frac{\alpha}{2}\right), \text { and } & V=\left(\frac{1}{2}-\frac{z}{h}\right)^{p}
\end{array}
$$

where $E c$ and $E m$ are the corresponding properties of the ceramic and metal, and $\rho$ density of material, respectively, and "P" is the volume fraction exponent which takes values greater than or equal to zero. The above power-law assumption reflects a simple rule of mixtures used to obtain the effective properties of the ceramic-metal plate. The rule of mixtures applies only to the thickness direction. Note that the volume fraction of the metal is high near the bottom surface of the plate, and that of the ceramic is high near the top surface. Furthermore, Eq. (1) indicates that the bottom surface of the plate $(\mathrm{z}=-\mathrm{h} / 2)$ is metal whereas the top surface $(\mathrm{z}=\mathrm{h} / 2)$ of the plate is ceramic.

In the present analysis, the shear deformation plate theory is suitable for the displacements (Merdaci et al [5]):

$$
\begin{gathered}
u(x, y, z)=u_{0}(x, y)-z \frac{\partial w_{b}}{\partial x}+\left(\frac{z}{2}\left(\frac{h^{2}}{4}-\frac{z^{2}}{3}\right)\right) \frac{\partial w_{s}}{\partial x} \\
v(x, y, z)=v_{0}(x, y)-z \frac{\partial w_{b}}{\partial y}+\left(\frac{z}{2}\left(\frac{h^{2}}{4}-\frac{z^{2}}{3}\right)\right) \frac{\partial w_{s}}{\partial y} \\
w(x, y, z)=w_{b}(x, y)+w_{s}(x, y)
\end{gathered}
$$

The stress-strain relations for a linear elastic plate and isotropic, are written in the following of

$$
\left\{\begin{array}{c}
\sigma_{x} \\
\sigma_{y} \\
\tau_{x y}
\end{array}\right\}=\left[\begin{array}{ccc}
Q_{11} & Q_{12} & 0 \\
Q_{12} & Q_{22} & 0 \\
0 & 0 & Q_{66}
\end{array}\right]\left\{\begin{array}{c}
\varepsilon_{x} \\
\varepsilon_{y} \\
\gamma_{x y}
\end{array}\right\}\left\{\begin{array}{c}
\tau_{y z} \\
\tau_{z x}
\end{array}\right\}=\left[\begin{array}{cc}
Q_{44} & 0 \\
0 & Q_{55}
\end{array}\right]\left\{\begin{array}{l}
\gamma_{y z} \\
\gamma_{z x}
\end{array}\right\}
$$

where $\left(\sigma_{x}, \sigma_{y}, \tau_{x y}, \tau_{y z}, \tau_{y x}\right)$ and $\left(\varepsilon_{x}, \varepsilon_{y}, \gamma_{x y}, \gamma_{y z}, \gamma_{y x}\right)$ are the stress and strain components, respectively. Using the material properties defined in Eq.(9), the stiffness coefficients, Qij, can be expressed as

$$
Q_{11}=Q_{22}=\frac{E(z)}{1-v^{2}}, Q_{12}=\frac{v E(z)}{1-v^{2}}, Q_{44}=Q_{55}=Q_{66}=\frac{E(z)}{2(1+v)},
$$

\section{THE SOLUTION METHOD}

The Hamilton's principle is used herein to derive the equations of motion. The principle can be stated in analytical from as Reddy [11].

$$
\int_{0}^{T}(\delta U+\delta V-\delta K) d t=0
$$

Where $\delta U$ : variation of strain energy; $\delta V$ : variation of potential energy; $\delta K$ : variation of kinetic energy. The variation of strain energy of the plate is calculated by

$$
\delta U=\int_{-h / 2}^{h / 2} \int_{A}\left[\sigma_{x} \delta \varepsilon_{x}+\sigma_{y} \delta \varepsilon_{y}+\tau_{x y} \delta \gamma_{x y}+\tau_{y z} \delta \gamma_{y z}+\tau_{x z} \delta \gamma_{x z}\right] d A d z
$$

The variation of potential energy of applied loads can be expressed as

$$
\delta V=-\int_{A} q\left(\delta w_{b}+\delta w s\right) d A
$$

The variation of kinetic energy of the plate can be written as

$$
\delta K=\int_{-h / 2}^{h / 2} \int_{A} \rho(z)(u \delta u+v \delta v+w \delta w) d A d z
$$

where dot-superscript convention indicates the differentiation with respect to the time variable t; and $\left(I_{1}, I_{2}, I_{3}, I_{4}, I_{5}, I_{6}\right)$ are mass inertias defined as 


$$
\left(I_{1}, I_{2}, I_{3}, I_{4}, I_{5}, I_{6}\right)=\int_{-h / 2}^{h / 2}\left(1, z, z^{2}, f(z), z f(z), f(z)^{2}\right) \rho(z) d z
$$

\section{ANALYTICAL SOLUTIONS FOR FG PLATES}

Rectangular plates are generally classified according to the type of support used. This paper is concerned with the exact solution for a simply supported FG plate. The following boundary conditions are imposed at the side edges:

$$
\begin{aligned}
& v_{0}=w_{b}=w_{s}=\frac{\partial w_{b}}{\partial y}=\frac{\partial w_{s}}{\partial y}=N_{x}=M_{x}^{b}=M_{x}^{s}=0 \text { and } x=0, a \\
& u_{0}=w_{b}=w_{s}=\frac{\partial w_{b}}{\partial x}=\frac{\partial w_{s}}{\partial x}=0, N_{y}=M_{y}^{b}=M_{y}^{s}=0 \text { and } y=0, b
\end{aligned}
$$

where $\boldsymbol{\lambda}=\boldsymbol{m} \boldsymbol{\pi} / \boldsymbol{a}$ and $\boldsymbol{\mu}=\boldsymbol{n} \boldsymbol{\pi} / \boldsymbol{b},{ }_{\text {}} \boldsymbol{m} »$ and ${ }_{«} \boldsymbol{n} »$ are mode numbers. For the case of a sinusoidally distributed load, we have

$$
m=n=1 \quad \text { and } \quad q_{11}=q_{0}
$$

Following the Navier solution procedure, we assume the following form of solution for (u,v,wb,ws) that satisfies the boundary conditions.

$$
\left\{\begin{array}{c}
u \\
v \\
w_{b} \\
w_{s}
\end{array}\right\}=\sum_{m=1}^{\infty} \sum_{n=1}^{\infty}\left\{\begin{array}{c}
U_{m n} e^{i \omega t} \cos (\lambda x) \sin (\mu y) \\
V_{m n} e^{i \omega t} \sin (\lambda x) \cos (\mu y) \\
W_{b m n} e^{i \omega t} \sin (\lambda x) \sin (\mu y) \\
W_{s m n} e^{i \omega t} \sin (\lambda x) \sin (\mu y)
\end{array}\right\},
$$

where Umn, Vmn,Wbmn, and Wsmn are arbitrary parameters. Can be combined into a system of equations as:

$$
\left([K]-\omega^{2}[M]\right)\{\Delta\}=\{0\},
$$

where $[\mathrm{K}]$ and $[\mathrm{M}]$, stiffness and mass matrices, respectively, and represented as:

$$
\left(\left[\begin{array}{llll}
a_{11} & a_{12} & a_{13} & a_{14} \\
a_{12} & a_{22} & a_{23} & a_{24} \\
a_{13} & a_{23} & a_{33} & a_{34} \\
a_{14} & a_{24} & a_{34} & a_{44}
\end{array}\right]-\omega^{2}\left[\begin{array}{cccc}
m_{11} & 0 & 0 & 0 \\
0 & m_{22} & 0 & 0 \\
0 & 0 & m_{33} & m_{34} \\
0 & 0 & m_{34} & m_{44}
\end{array}\right]\right)\left\{\begin{array}{c}
U_{m n} \\
V_{m n} \\
W_{b m n} \\
W_{s m n}
\end{array}\right\}=\left\{\begin{array}{c}
0 \\
0 \\
q_{m n} \\
0
\end{array}\right\}
$$

\section{RESULTS AND DISCUSSIONS}

In this section various numerical examples are presented and discussed to verify the accuracy of the present theory in predicting the free vibration responses of simply supported FG plates. One type of $\mathrm{FG}$ plates of $\mathrm{Al} / \mathrm{ZrO}_{2}$ are used in this study. The material properties of FG plates are listed in Table 1. Unless otherwise has been stated, the following relations have been used for presentations of non-dimensional natural frequencies.

Table 1. Material properties used in FG plate

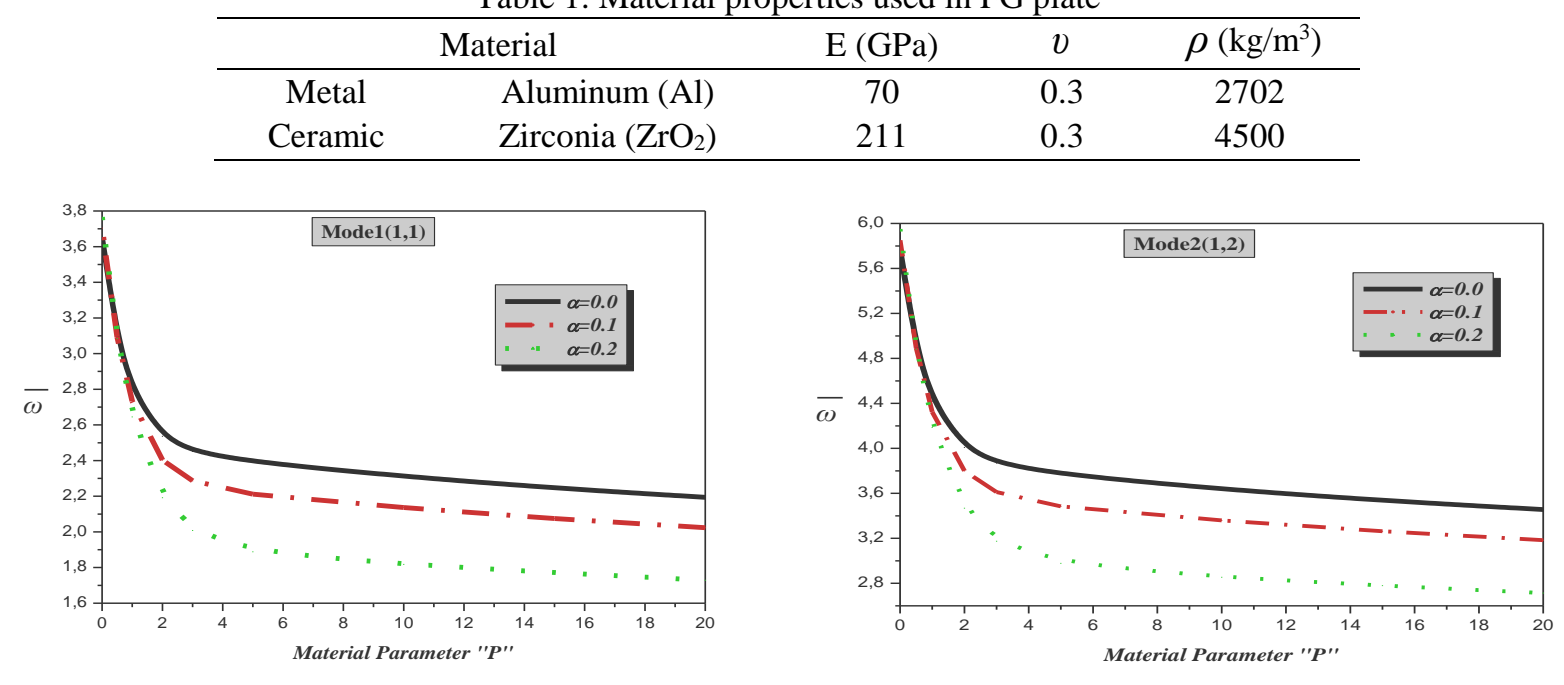



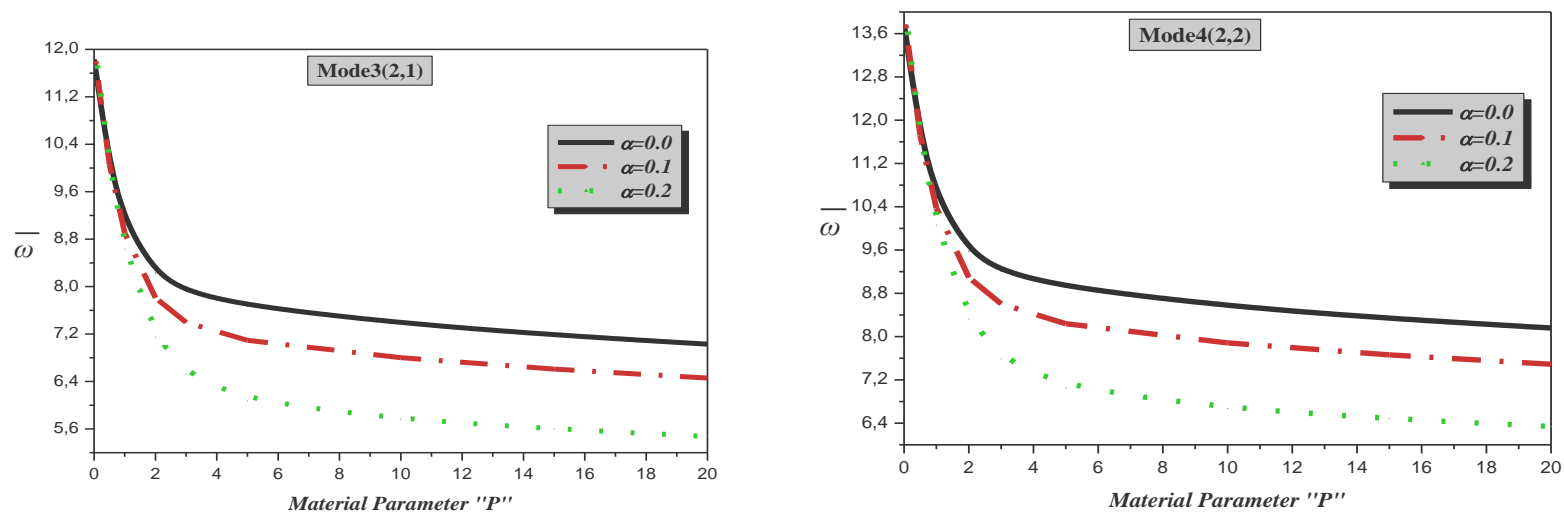

Fig.2. Fundamental frequency of plate porous according to the material power index $P$.

From the curves presented in Figs.2, it can be noted that more than the parameter of the power index of the material "P" is high, more than the fundamental frequency in the porous $(\alpha=0.1$ and 0.2$)$ and non-porous $(\alpha=0.0)$ plate FG structures is decreased, which whatever the number of waves. However, increasing the porosity factor causes an increase in the frequency. Therefore, the maximum frequency is obtained for a ceramic plate $(\mathrm{P}=0)$ and a porosity factor $\alpha=0.2$.
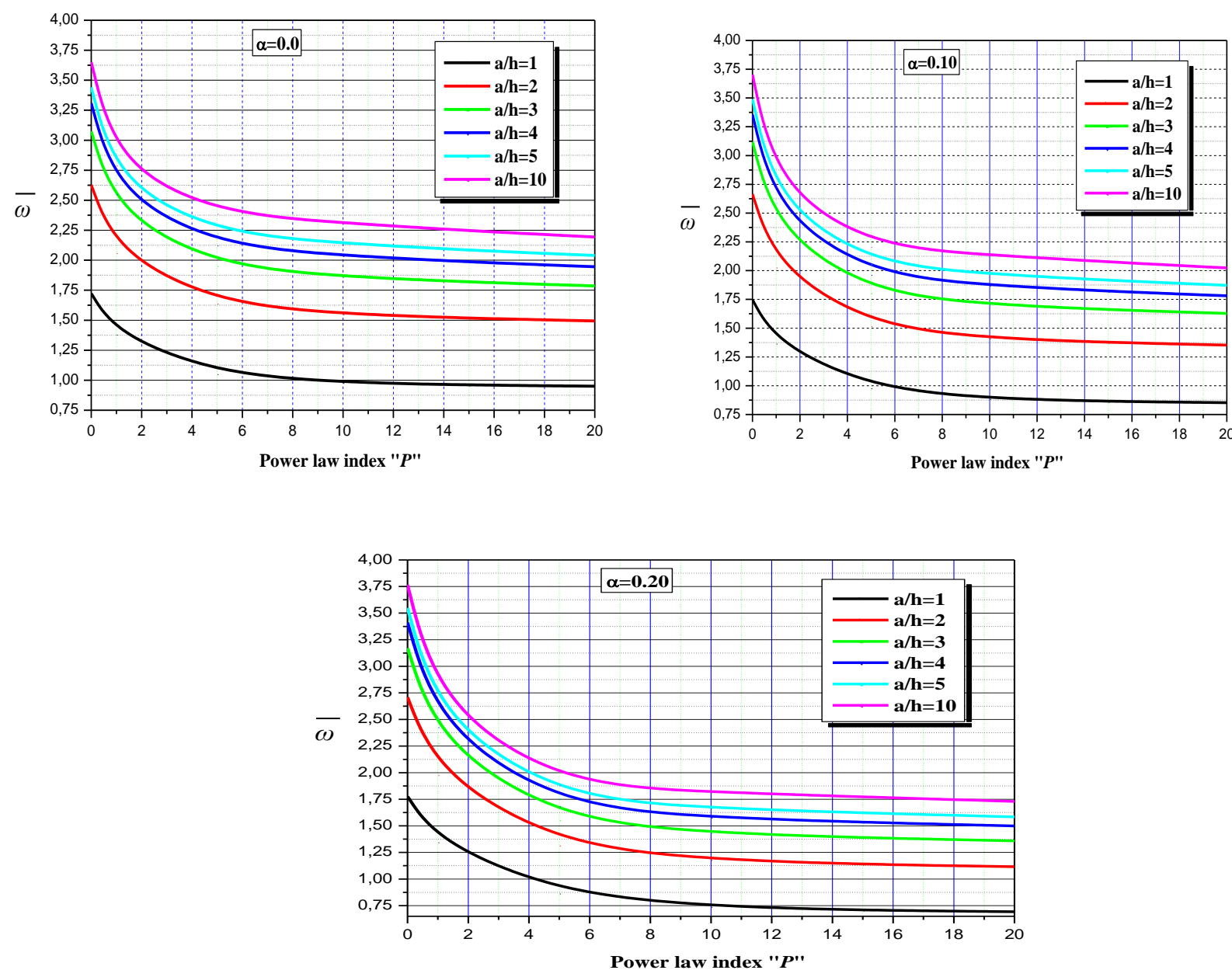

Fig.3. Non-dimensional fundamental natural frequency of simply supported FG rectangular plates as function of power law index $P$ for different a=h and different porosity factor $\alpha$. 
The non-dimensional fundamental natural frequency $\mathrm{v}$ of simply supported rectangular FG plates $(b=2 a)$ and power law index $\mathrm{P}$ for various values of side-to-thickness ratios $(\mathrm{a}=\mathrm{h})$ and different porosity factor $(\alpha=0.0 ; 0.10$ and 0.20 ) are plotted in Figs.3 based on the present high order shear deformation theory. As shown, the frequency decreases significantly with the increase of $\mathrm{P}$.

\section{CONCLUSION}

In this work, the analysis of the free vibration of porous plates FG is examined by a new simple theory of high order shear deformation. This theory satisfies the nullity of the stresses at the upper and lower surfaces of the plate without using the shear correction factor. The law of the modified mixture covering the porosity phases is used to roughly describe the properties of plate FG with porosity. On the basis of the present theory of the plate, the equations of motion are derived from the principle of virtual works and the principle of Hamilton. From this work, we can say that the present theory of plate FG proposed is accurate and simple for the resolution of the mechanical behavior of FG plates with porosity.

\section{REFERENCES}

[1] Koizumi M. FGM activities in Japan[J]. Composites;28(1-2):14(1997).

[2] Zhu, J. Lai, Z. Yin, Z. Jeon, J. and Lee, S. Fabrication of ZrO2$\mathrm{NiCr}$ functionally graded material by powder metallurgy. Mater. Chem. Phys, 68(1-3), 130-135, (2001).
[3] Wattanasakulpong, N. Prusty, B.G. Kelly, D.W. and Hoffman, M. Free vibration analysis of layered functionally graded beams with experimental validation. Mater. Des, 36, 182-190, (2012).

[4] Wattanasakulpong, N. and Ungbhakorn, V. Linear and nonlinear vibration analysis of elastically restrained ends FGM beams with porosities. Aerosp.Sci. Technol, 32(1),111-120, (2014).

[5] S. Merdaci, A. Tounsi, M.S.A. Houari, I. Mechab, H. Hebali, S. Benyoucef, Two new refined shear displacement models for functionally graded sandwich plates, Arch. Appl. Mech. 81 1507e22. (2011)

[6] S.Merdaci, A. Tounsi, A.Bakora " A novel four variable refined plate theory for laminated composite plates" ; An International Journal Steel \& Composite Structures; N.4, Vol 22, pp 713 732,(2016).

[7] MERDACI Slimane "Study and Comparison of Different Plate Theory"; International Journal of Engineering Research And Advanced Technology (IJERAT); Vol.3 (Number 8), pp 49-59, (2017).

[8] A. Hadj Mostefa, S.Merdaci, and N. Mahmoudi "An Overview of Functionally Graded Materials «FGM»", Proceedings of the Third International Symposium on Materials and Sustainable Development, ISBN 978-3-319-89706-6, pp. 267-278, (2018).

[9] Merdaci Slimane "Analysis of Bending of Ceramic-Metal Functionally Graded Plates with Porosities Using of High Order Shear Theory"; Advanced Engineering Forum; Vol.30, pp 54-70, (2018).

[10] Merdaci .S, Belghoul.H, "High Order Shear Theory for Static Analysis Functionally Graded Plates with Porosities", Comptes rendus Mecanique, Vol 347, Issue3, pp 207-217, (2019).

[11] Reddy, J. N. Energy principles and variational methods in applied mechanics, Wiley, New York. 406.(2002).

[12] Reddy, J. N. "A simple higher-order theory for laminated composite plates." J. Appl. Mech., 51(4), 745. (1984).

[13] Reddy, J. N. "Analysis of functionally graded plates." Int. J. Numer. Methods Eng., 47, 663-684. 404.(2000). 